\title{
1 Sharing the right data right: a symbiosis with machine 2 learning
}

3 Sotirios A. Tsaftaris ${ }^{1, *}$, and Hanno Scharr ${ }^{2}$

$4{ }^{1}$ Institute for Digital Communications, School of Engineering, University of Edinburgh,

5 Edinburgh, EH9 3FG, UK

$6 \quad{ }^{2}$ Institute of Bio- and Geosciences: Plant Sciences (IBG-2) Forschungszentrum Jülich GmbH, D-

752425 , Jülich, Germany

$8 \quad{ }^{*}$ Correspondence: S.Tsaftaris@ed.ac.uk (S.A. Tsaftaris), URL: http://tsaftaris.com; h.scharr@fz-

9 juelich.de (H. Scharr), URL: http://www.fz-juelich.de/ibg/ibg-

$10 \quad$ 2/EN/Staff/Enabling\%20Technologies/Scharr_Hanno/Scharr.html

12 Keywords: open data, machine learning, plant phenotyping

\section{Abstract}

15 In 2014 plant phenotyping research was not benefiting from the machine learning revolution as

16 appropriate data were lacking. We report the success of the first open dataset in image-based

17 plant phenotyping suitable for machine learning, fuelling a true interdisciplinary symbiosis,

18 increased awareness and steep performance improvements in key phenotyping tasks.

\section{Advancing plant phenotyping by sharing 'problems'}

22 Appropriate training and testing data are at the heart of computer vision (CV) and machine

23 learning (ML) research as means for developing and evaluating novel approaches. However, in

24 2014, appropriate data for image-based phenotyping problems were lacking. Thus, plant

25 phenotyping was not benefiting from this data-driven revolution [1] and CV/ML researchers

26 were largely unaware of phenotyping applications. To address these limitations, we opened a

27 collection of plant data for several phenotyping tasks including two 'hot' CV/ML problems: leaf

28 segmentation a 'multi-instance segmentation' problem and leaf counting an 'object counting' 
1 problem [2,3]. Our objectives were to measure how well broad ML algorithms could solve major

2 phenotyping tasks but also enlarge the community of scientists considering phenotyping

3 applications. Within four years our datasets became very popular, even reaching 'standard

4 dataset' status for multi-instance segmentation and object counting. Most importantly we saw

5 tremendous improvement in performance in solving these two tasks. Here, we report on our

6 efforts to promote the dataset, which we consider were vital for its success and aim to offer

7 advice on making problems interesting and corresponding data useful for the CV/ML

8 community.

10 Setting the problem: the competitions

11 The current race for publications in ML and CV is mostly won by the 'best performing' solution

12 and we wanted to leverage this to maximise attention to plant phenotyping problems. We, thus,

13 organised a 'competition' (typically in the ML community refer to as 'challenge') as a rapid and

14 visible publication route to attract CV/ML researchers. Prerequisite for a successful challenge is a

15 challenging but doable problem, not too hard and not yet solved. Leaf segmentation and counting

16 are ideal problems as they were amenable to several approaches and can be understood by

17 everyone. As clarity is mission critical, clear and easy to interpret performance measures are a

18 must. This is trivial for counting, where we decided to use average absolute count difference and

19 average count difference. Evaluating multi-instance segmentation results is less simple. Suitable

20 measures for single instance segmentation are well established e.g. the 'intersection of the union'

21 score or the very similar and classical Dice score. Both range between zero and one and are thus

22 easily interpretable as success rates being perfect at $100 \%$. Since no well-established criteria

23 existed when we established the competition, we introduced a multi-instance version of the Dice

24 score. We ensured that neither reordering of instances nor exchanging roles of ground truth and

25 test solutions have an influence on the result. The resulting 'Symmetric best Dice' measure is

26 thus as easily interpretable as the single instance version. We made participants' lives as easy as

27 we could: accompanying our datasets were scripts to load and pre-process data and code

28 evaluating the proposed metrics for measuring performance. In combination, our strategy not

29 only lowered the barrier to entry but also standardized the results and their presentation

30 ultimately allowing direct comparison of methods (see Box 1). 


\section{Setting the stage: the workshops}

3 To incentivise participation, we organized the first challenge in 2014 as part of a workshop

4 accepting also full-length papers describing challenge submissions. To make this publication

5 avenue as attractive as possible we organized the workshop together with an internationally

6 renowned and high-ranking computer vision conference to maximise visibility. In addition, full-

7 length papers were published together with main conference proceedings [4] -a great value for

8 CV researchers- and extended versions were bundled in a special issue [5] of a computer vision 9 journal.

10 As a community building measure and to promote application-related problems, the

11 workshop also accepted 'problem statement' papers. These papers do not describe solutions, as is

12 common for CV/ML conferences, but properly describe unsolved, but relevant, problems in an

13 application area. Thus, application scientists, experts in these problems, were engaged without

14 being experts in CV or ML.

15 This workshop format was so well received across the disciplines that we proceeded to

16 organize it almost yearly in conjunction with top CV conferences (e.g. ICCV, ECCV, and BMVC 17 (twice)).

\section{Setting the baseline: the collation study}

20 We compiled a collation study summarizing the results, where all challenge contributors served 21 as co-authors [6]. This showed the quality bandwidth of the applied solutions, but also set the 22 baseline for comparing future approaches.

\section{Releasing the data: the paper and the website}

25 Given the success of the first workshop and challenge we decided to publish a paper describing,

26 for the first time, a large collection of image analysis problems that arise in plant phenotyping;

27 and to offer accompanying data, performance metrics and several baseline methods

28 demonstrating the challenging aspect of the data. This coincided with the continuous requests

29 from colleagues for the challenge data and to perform evaluations on the test set. Together with

30 the release of the paper [3], we therefore created a website ${ }^{\mathrm{ii}}$ allowing the download of data after 
1 registration. This registration information allows us to track the success and impact of the dataset

2 (see Figure 1B-D).

\section{The impact}

5 - As of September 2018, 1600 requests were recorded, with an overall exponential growth

\section{What's next}

28 These remarkable gains are mostly seen for Arabidopsis wild-type where we had released

29 adequate number of training data. We should now collectively focus to translate such 
However, when do we stop and what do we have to do to get there? Typically, in CV/ML

2 a limit is met when automated algorithms achieve or surpass human level performance. For leaf

3 counting, a recent study showed that 0.29 is the current human expert performance [9], clearly,

4 we are not there yet but coming closer. For leaf segmentation such study is currently lacking as

5 is tedious to perform. Deep learning approaches need more annotated data to help improve

6 performance yet obtaining annotated data of significant variability and size is difficult. Options

7 to synthesise data [10-12] or use citizen scientist platforms to collect annotations can help

8 increase scalability and is an area of active research and activity [9].

9 To enable continuous evaluation, we have now set up an online system ${ }^{\text {iv }}$ that evaluates

10 performance of approaches based on a held-out testing set. The ideal would be to use a sandbox

11 virtual system that executes code on a dedicated server, but this requires dedicated funding.

12 Finally, to improve performance on other cultivars, plants and tasks, appropriate training data are

13 necessary which the community together should provide (see Box 1 for advice).

\section{Conclusion}

16 We want to emphasize that opening data is good practice as it allows reproducibility of science.

17 In the plant sciences this is starting to take place. Yet we still hesitate to publish data when we

18 don't have a good solution. This article demonstrates that potential for impact exists even if we

19 do not yet have the perfect solution -in fact it is better to show the limitations of current

20 approaches and demonstrate difficulty. If we open our data the 'right' way, the CV/ML

21 community can and will solve our problems 'for free'-a true symbiosis.

\section{Resources}

24 i. http://www.plant-phenotyping.org/CVPPP2014

25 ii. https://www.plant-phenotyping.org/datasets

26 iii. https://www.plant-phenotyping.org/datasets-impact

27 iv. https://competitions.codalab.org/competitions/18405

28 v. https://zenodo.org/ 


\section{References}

2 [1] Minervini, M. et al. (2015) Image analysis: The new bottleneck in plant phenotyping

3 [applications corner]. IEEE Signal Processing Magazine 32, pp. 126-131.

4 [2] Scharr, H. et al. (2014) Annotated Image Datasets of Rosette Plants, Forschungszentrum

5 Jülich, Report No.: FZJ-2014-03837, pp. 1-16

6 [3] Minervini, M. et al. (2016) Finely-grained annotated datasets for image-based plant

7 phenotyping. Pattern Recognition Letters 81, pp. 80-89

8 [4] Agapito, L. et al. (eds.) (2015) Computer Vision-ECCV 2014 Workshops, Zurich,

9 Switzerland, September 6-7 and 12, 2014, Proceedings, Part IV, Springer International

10 Publishing, Vol. 8928, 396 pages

11 [5] Scharr, H. et al. (2016) Special issue on computer vision and image analysis in plant

12 phenotyping. Machine Vision and Applications 27, pp. 607-609

13 [6] Scharr, H. et al. (2016) Leaf segmentation in plant phenotyping: A collation study.

14 Machine Vision and Applications 27, pp. 585-606. Special Issue on Computer Vision and Image

15 Analysis in Plant Phenotyping.

16 [7] Romera-Paredes, B. and Torr, P. H. S. (2016) Recurrent Instance Segmentation.

17 Proceedings of European Conference on Computer Vision, pp 312-329. First version: Arxiv

18 (2015) https://arxiv.org/abs/1511.08250

19 [8] Ren, M. and Zemel, R. S. (2017) End-To-End Instance Segmentation With Recurrent

20 Attention. The IEEE Conference on Computer Vision and Pattern Recognition (CVPR), pp.

$21 \quad 6656-6664$

22 [9] Giuffrida, M. V. et al. (2018) Citizen crowds and experts: observer variability in image-

23 based plant phenotyping. Plant methods 14, pp. 1-14.

24 [10] Giuffrida, M. V. et al. (2017) ARIGAN: Synthetic arabidopsis plants using generative

25 adversarial network. Proceedings of the 2017 IEEE International Conference on Computer

26 Vision Workshop (ICCVW), Venice, Italy, pp. 22-29.

27 [11] Zhu, Y. et al. (2018) Data Augmentation using Conditional Generative Adversarial

28 Networks for Leaf Counting in Arabidopsis Plants. Computer Vision Problems in Plant

29 Phenotyping (CVPPP2018) pp. 1-11.

30 [12] Ward, D. et al. (2018) Deep Leaf Segmentation Using Synthetic Data. Computer Vision

31 Problems in Plant Phenotyping (CVPPP2018) pp. 1-13. 
1 Figure 1. (A) An example of the content and annotations available in the dataset (figure adapted 2 and modified from [3]); (B) Cumulative download requests since release (December 2015, axis dataset release refer to challenge contributions.

\section{Box 1. How to share data successfully by making it useful for others}

- Open the RIGHT data for the RIGHT problem (neither undoable nor solved): Publish data

1

$$
\text { not just as means of verification but as "problem statements" [2,3] and give a baseline [6]. }
$$

$$
\text { Observe the balance: if the baseline performs already too well, it is not a challenging }
$$
problem.

- Appropriate data for the problem: Data without accompanying annotations are not very

useful for the machine learning era of today.

- Use terminology attractive for the intended audience: For example, leaf segmentation is a 17 multi-instance object segmentation.

- Adhere to Findable, Accessible, Interoperable, and Re-usable (FAIR) principles: We opened the data, described their origin, metadata, how to read/write, and how to share findings.

- Decide on suitable metrics: Ideally a single, well established, and easy to interpret measure should be provided to evaluate performance.

- Offer implementations of error metrics and keep it standardised: Open training data 
1 Figure I. How users heard about the data, shown as percentage over three periods. The first four 2 options correspond to actions that we had direct influence over, whereas the rest were out of our 3 hands. PRL paper: We observe that publishing a tech report [2] satisfies the FAIR data but is 4 just an entry point. A peer-reviewed paper gave us better visibility [3]. Challenges: We

5 organised workshops and challenges, were inclusive and rallied the community with the collation 6 study paper [6]. Invitation: We directly emailed people after building our own contact list and 7 ensured that the data are listed in relevant databases (Lists). This is what fed the initial growth, 8 which of course changed completely as more people discovered the data as soon as the first 9 "high-impact" computer vision paper cited the dataset. Then growth over the years was largely 10 fuelled by papers citing the PRL paper [3] and of course by search engines, so having a 11 website helps immensely. (The search engine numbers could be inflated as search may have been 12 the means and not the origin.) 


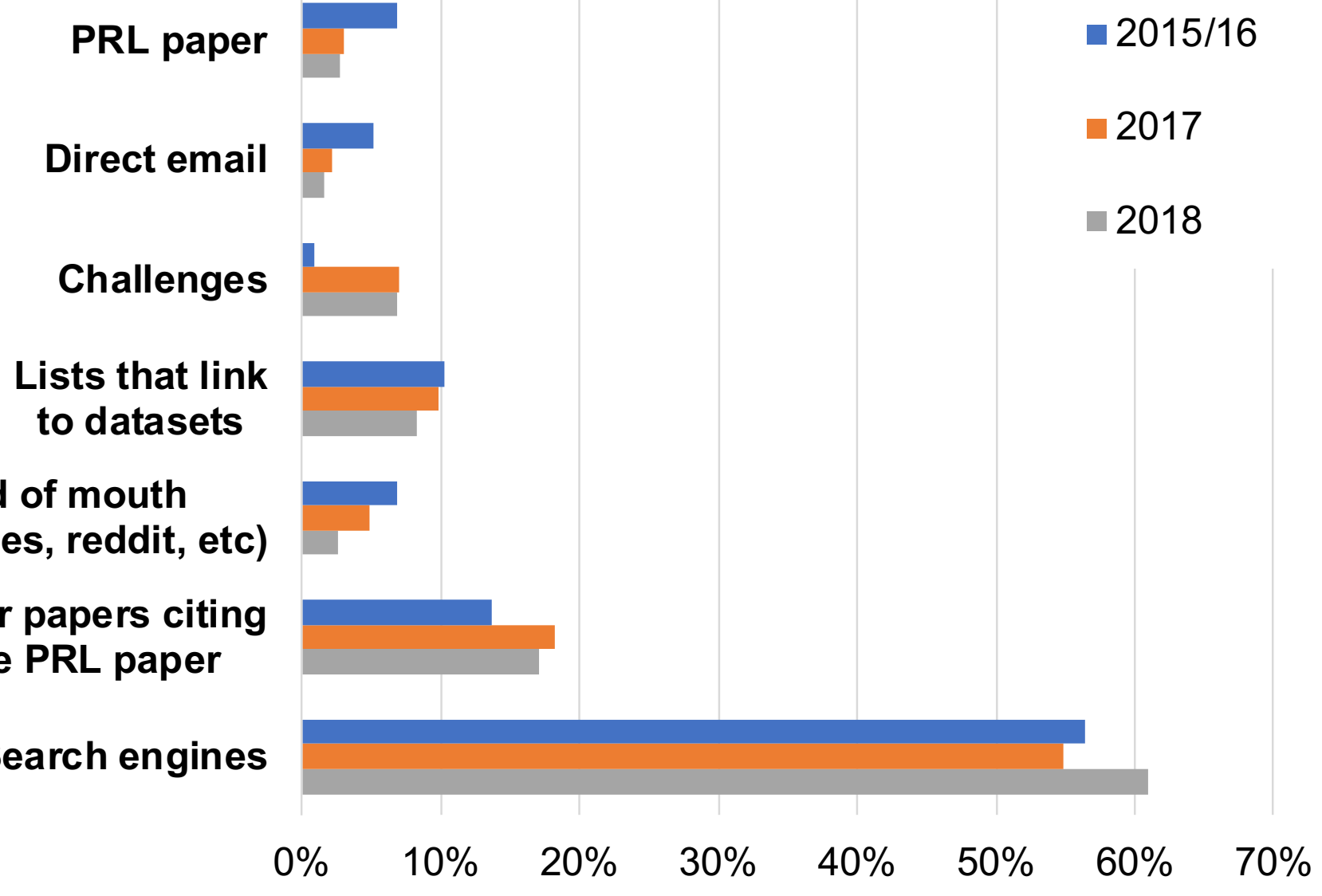


(B) Dommiosts over time
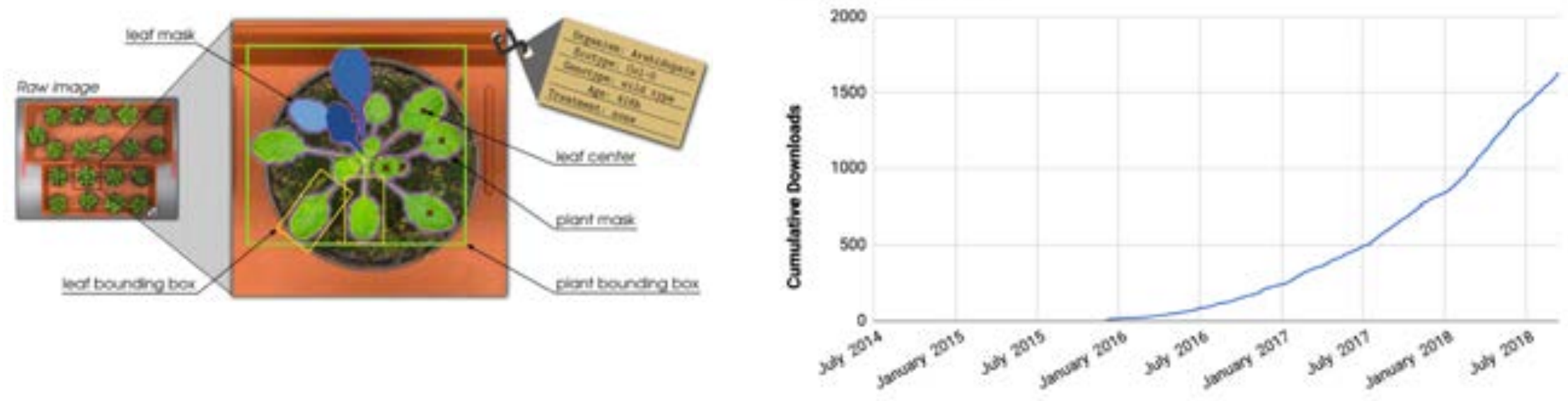

(C) Bactgonond atuen

\section{(D) Performance over time}

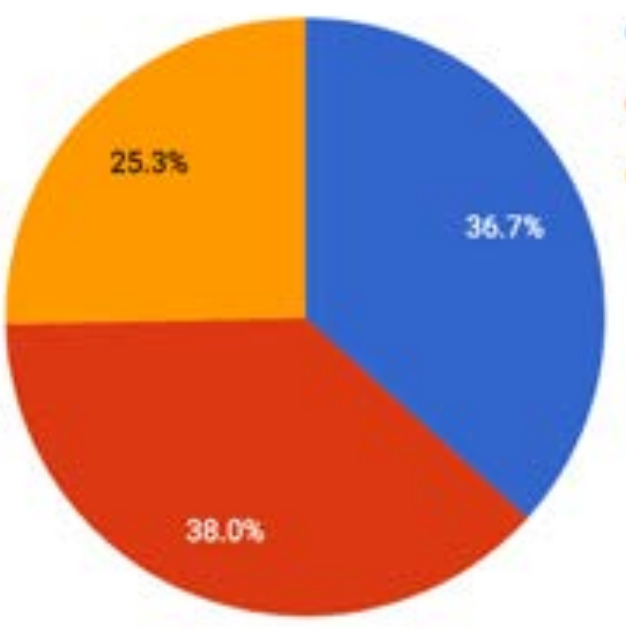

- Vision scientist [plants applications] - Vision scientist [other applications] - Other scientist

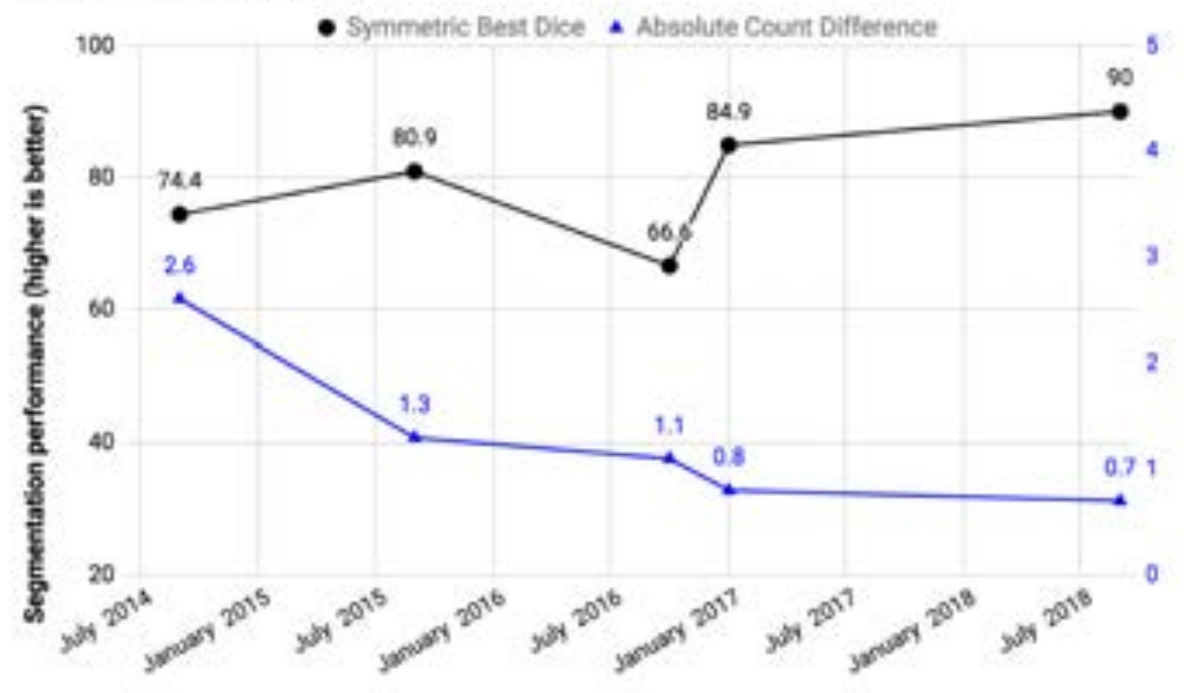

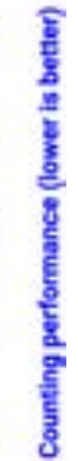

